\title{
Anti-Nociceptive Effects of Elcatonin Injection for Postmenopausal Women with Back Pain: A Randomized Controlled Trial
}

\author{
Shota Ikegami ${ }^{*}$, , Mikio Kamimura ${ }^{2}$, Shigeharu Uchiyama ${ }^{1}$, Hiroyuki Nakagawa ${ }^{3}$, Hiroyuki Hashidate ${ }^{1}$, \\ Kenji Takahara ${ }^{4}$, Jun Takahashi ${ }^{1}$ and Hiroyuki Kato ${ }^{1}$
}

\author{
${ }^{1}$ Department of Orthopaedic Surgery, Shinshu University School of Medicine, 3-1-1 Asahi, Matsumoto 390-8621, Japan \\ ${ }^{2}$ Center for Osteoporosis and Spinal Disorders, Kamimura Orthopaedic Clinic, Matsumoto 399-0021, Japan \\ ${ }^{3}$ Department of Orthopaedic Surgery, Suwa Red Cross Hospital, Suwa 392-8510, Japan \\ ${ }^{4}$ Department of Orthopaedic Surgery, Ina Central Hospital, Ina 396-8555, Japan
}

\begin{abstract}
Background: Eel calcitonin (elcatonin) injection is widely used for elderly patients suffering from somatic pain in Japan. However, there have been few reports on the analgesic effects of elcatonin injection. The purpose of this study was to examine the analgesic effects of elcatonin injection in postmenopausal women with lower back pain.

Methods: This study was designed as a double-blind, randomized, placebo-controlled study. Thirty-six women aged $\geq 50$ years with acute lower back pain participated in this study. They were randomly divided into two treatment groups according to whether they received a placebo or a weekly trigger point injection of elcatonin (20 units). They were observed for 5 weeks and the extent of pain at motion and at rest according to the visual analog scale (VAS) was evaluated. The mean VAS scores for the elcatonin group were then compared with those of the placebo group.

Results: There were no statistically significant differences in the mean VAS scores for pain at rest between the two groups during the 5-week treatment course. However, the mean VAS scores for motion pain in the elcatonin group were significantly lower than those in the placebo group at the third, fifth and sixth weeks.

Conclusions: Elcatonin injection (20 units) significantly relieved motion pain in the lower back in postmenopausal women after three weeks of treatment. This analgesic effect continued for the subsequent 3 weeks.
\end{abstract}

Keywords: Postmenopause, back pain, anti-nociceptive.

\section{INTRODUCTION}

The frequency of hospital visits by elderly patients is apparently increasing. Among these patients, lower back pain is a relatively common complaint.

Calcitonin is a polypeptide hormone secreted from the parafollicular $\mathrm{C}$ cells of the thyroid gland $[1,2]$. This hormone is involved in the regulation of calcium homeostasis in blood and its metabolism in bone [3]. Repeated intramuscular or intravenous injection of calcitonin alleviates pain related to osteoporosis [4], and it is widely used in clinical practice as a treatment for elderly patients.

A previous meta-analysis confirmed the analgesic effects of salmon calcitonin (100 to $200 \mathrm{IU}$ ), which is administered either intranasally or intramuscularly, on lower back pain associated with osteoporotic vertebral compression fracture [5]. However, such high doses of calcitonin are not sanctioned in Japan. Instead, elcatonin (20 units), which is a calcitonin intramuscular injection, has been widely used. However, there are few reports on the analgesic effects of elcatonin injection [6].

*Address correspondence to this author at the Department of Orthopaedic Surgery, Shinshu University School of Medicine, Asahi 3-1-1, Matsumoto, Nagano 390-8621, Japan; Tel: +81-263-37-2659; Fax: +81-263-35-8844; E-mail: sh.ikegami@gmail.com
Postomenopausal women visit our institution for lower back pain and are regularly treated with elcatonin injection therapy. However, as the evidence for this therapy is insufficient, we decided to investigate its efficacy in clinical practice. We hypothesized that elcatonin injection treatment is an effective method for the relief of lower back pain. We therefore examined the analgesic effects of elcatonin $(20$ units) treatment in postmenopausal women in regard to resting pain and motion pain in the lower back in a 2-year, double-blind, randomized, placebo-controlled study.

\section{MATERIALS AND METHODOLOGY}

\section{Subjects}

Women aged $\geq 50$ years with acute lower back pain and who visited to Suwa Red Cross Hospital from August 2001 to March 2003 were enrolled in this study. The inclusion and exclusion criteria are listed (Table 1). Patients who were not able to sit up because of severe back pain, and suspected to have a fresh vertebral fracture, were excluded from the study. Whether they had any vertebral fracture or not was determined using standard anteroposterior and lateral radiographs of the spine in the standing position. The study was approved by the Ethics Committee of Suwa Red Cross Hospital. We explained the contents of the study to the patients who satisfied the inclusion criteria, and those providing informed consent were enrolled as study subjects. 
Table 1. Inclusion and Exclusion Criteria for the Trial

\begin{tabular}{|c|c|}
\hline Inclusion Criteria & Exclusion Criteria \\
\hline \hline Postmenopausal woman & Premenopausal woman \\
\hline Pain, primarily in lower back & Pain at several sites, e.g., buttock, thigh \\
\hline Able to regularly visit hospital & Bedridden due to severe pain \\
\hline Acute moderate back pain including unrecognizable vertebral fractures by X-Ray & Obvious fresh vertebral fracture, as diagnosed by X-Ray \\
\hline & Prior use of elcatonin \\
\hline & Malignancy \\
\hline
\end{tabular}

\section{Randomization and Clinical Intervention}

When the participants first visited our hospital, we used the envelope method to randomly divide them into the elcatonin group and the placebo group. The elcatonin group was given a trigger point injection of a mixture of elcatonin (20 units) and $1 \%$ lidocaine hydrochloride $(8 \mathrm{ml})$. The placebo group was given trigger point injection of a mixture of the placebo (1\% lidocaine hydrochloride $[1 \mathrm{ml}])$ and $1 \%$ lidocaine hydrochloride $(8 \mathrm{ml})$. The patients and operators were blinded in regard to the medicines injected. The injections were repeated once weekly until pain relief. We entrusted the timing of injection termination to each participant. Participants had regularly taken nonsteroidal anti-inflammatory drugs (etodolac, $100 \mathrm{mg}$ ) three times per day until sufficient relief of their pain. They also regularly took active vitamin D3 and calcium everyday throughout the study period.

\section{Measurement Variables}

Participants' lower back pain was classified as "resting pain" or "motion pain". Resting pain meant the pain caused by keeping the recumbent, sitting or standing position. Motion pain meant the pain caused by changing the posture, for example, recumbent to sitting or sitting to standing position. However, it may be difficult to distinguish these pains accurately. Therefore, we asked patients to determine which type of pain they had. Participants were ordered to report their resting pain and motion pain weekly using the visual analog scale (VAS) as well as the amount of NSAIDs taken during the previous week. We evaluated each participant from the week of her first visit until the sixth week (for 5 weeks). We also evaluated the total injection volume and the amount of NSAIDs used by each participant. We measured the bone mineral density (BMD) at the posteroanterior spine (L2-4) and biochemical bone markers (serum levels of bone alkaline phosphatase [BAP] and urine levels of cross-linked N-telopeptides of type I collagen [NTx]) as baseline data for the participants at the time of their first visit to our hospital. BMD was measured with a dual-energy X-Ray absorptiometry (DXA) fan-beam bone densitometer (Hologic QDR 2000; Hologic Waltham, MA).

\section{Data Analysis}

We excluded inappropriate cases prior to performing the analysis. We assessed the differences in the mean VAS scores between the elcatonin group and the placebo group in regard to both resting pain and motion pain. We performed an analysis of variance with repeated measures to determine the differences between the two groups and over time. If a significant difference was detected between the elcatonin group and the placebo group by the analysis, we performed Welch's $t$ test between the two groups regarding the mean VAS scores for each week. We also assessed the differences between the means in baseline data (age, BMD, serum BAP, and urinary NTx) in each group by Welch's t test. We considered $p<0.05$ to indicate significance in all analyses. The statistical analysis was performed using SPSS for Windows 17.0 software program (SPSS, Chicago, IL, USA).

\section{RESULTS}

Fig. (1) shows the flow diagram throughout the trial. Thirty-six patients participated in the trial, and four of these were excluded before opening the study key. Seventeen patients were in the elcatonin group and 15 patients were in the placebo group.

Of the excluded participants, three were assigned to the elcatonin group and one was assigned to the placebo group. In the elcatonin group, two participants did not attend a follow-up on the second week, and one participant was not able to continue with the intervention beyond the third week. One of the participants missing the follow-up canceled further visits to our department because of adequate pain relief at 2 days after the first injection. The other patient was admitted to the hospital after 1 week with an eye disease that was unrelated to the study intervention, although she stated that she experienced sufficient pain relief to go on a trip after the first injection. The participant who was unable to continue intervention suffered pain and muscular weakness in her left leg at 2 weeks after the first injection and was admitted to our department. She has since received conservative treatment under a diagnosis of lumbar spinal canal stenosis and has since achieved sufficient pain relief. In the placebo group, only one participant failed to attend follow-up on the fourth week, having decided to forego further participation in the study. However, this patient also reported a gradual decrease in pain.

There were no significant differences in the mean age, BMD or biochemical bone markers between the two groups at the first visit (Table 2). The number of clinical interventions did not significantly differ between the elcatonin and placebo groups. The mean number of injections was $2.5 \pm 0.2$ (mean \pm standard error) in the elcatonin group, and $3.1 \pm 0.5$ in the placebo group, although this difference was not significant $(p=0.24,95 \%$ confidence interval $[95 \% \mathrm{CI}]-1.7,-0.4)$. In addition, there were no significant differences in the mean amount of NSAIDs used 


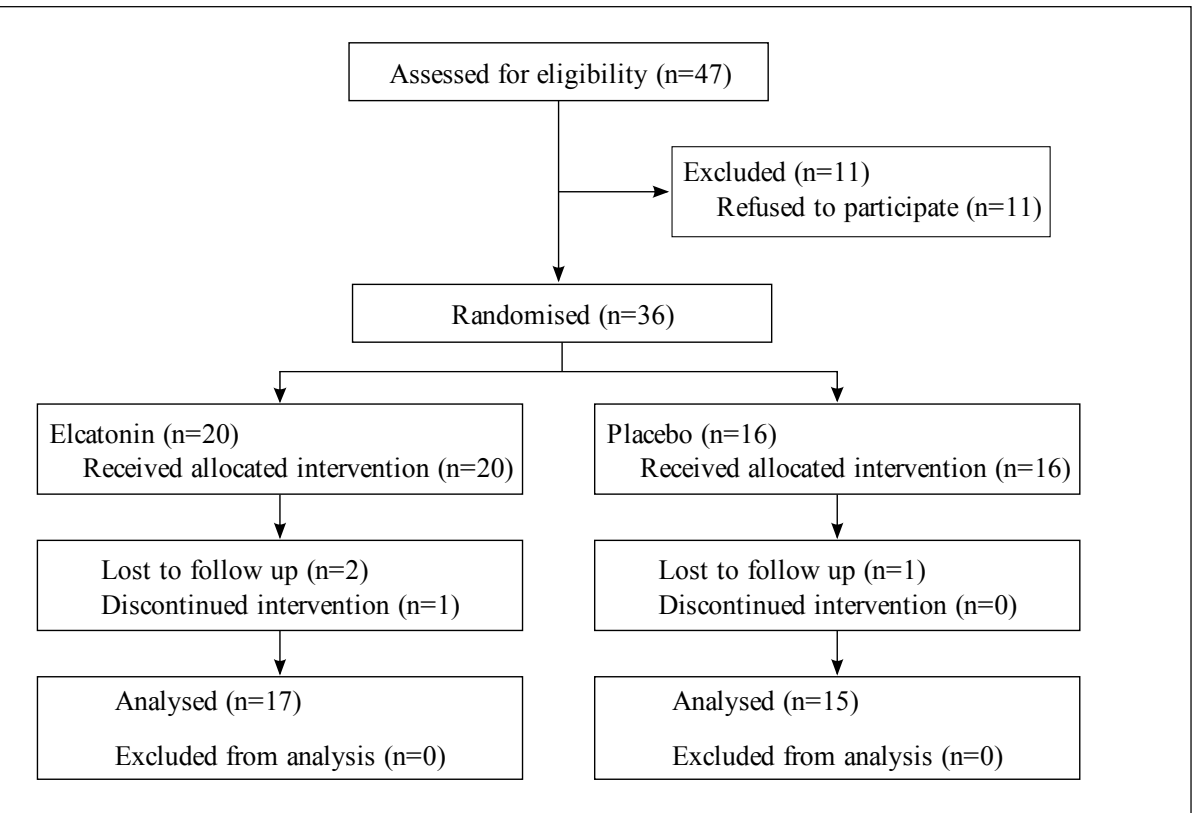

Fig. (1). Flow diagram showing the flow of participants through the trial.

between the two groups $(99.0 \pm 3.9$ tablets in the elcatonin group, $84.0 \pm 8.2$ tablets in the placebo group; $p=0.11,95 \%$ CI $3.9,33.9)$.

The patterns of pain at rest were very similar in the elcatonin and placebo groups (Fig. 2). The mean VAS scores were roughly 3.0 at the time of the first visit in each group $(3.00 \pm 2.60$ in the elcatonin group, $2.83 \pm 2.65$ in the placebo group), and these values decreased over time, reaching nearly 0 in the fifth week $(0.20 \pm 0.35$ in the elcatonin group, $0.34 \pm 0.59$ in the placebo group). There was a significant effect of the passage of time after the first visit, but there were no significant effect in regard to the type of injected drugs and no significant interaction between the drugs and the course of time (Table 3). In contrast, the course and extent of motion pain were not similar between the two groups. The mean VAS scores differed between the two groups, although the values in both groups decreased over time (Fig. 2). There were significant effects in regard to the time and the type of drug, but there was no significant interaction between these two factors (Table 3). Although the mean VAS scores of the two groups were similar at the time of first visit $(6.91 \pm 1.93$ in the elcatonin group, $7.04 \pm$ 2.44 in the placebo group), the mean VAS score of the elcatonin group decreased to a greater degree than that of the placebo group during the evaluation period. There was a statistically significant difference in the mean VAS scores between the two groups at the third $(p=0.047)$, fifth $(p=$ $0.047)$ and sixth weeks $(p=0.023)$. No adverse events were observed during the course of the trial.

\section{DISCUSSION}

We observed that the injection of elcatonin (20 units) was more effective at relieving lower back motion pain than a placebo in postmenopausal women; however, elcatonin was not significantly more effective at relieving pain at rest as compared with a placebo. Postmenopausal women tend to have lower back pain, particularly during motion, which often disturbs their activities of daily living. An injection of elcatonin (20 units) is useful for the relief of this type of pain.

Menopause is known to be a cause of osteoporosis in humans. Postmenopausal women often suffer from pain in their daily lives. One of the most important changes following menopause is the depletion of estrogen, which regulates gene expression. The depletion of this hormone influences the amount of gene products, including receptors and peptides (e.g. calcitonin) required for the modulation of nociceptive transmission. Accordingly, the depletion of

Table 2. Baseline Data for the Elcatonin and Placebo Groups

\begin{tabular}{|c|c|c|c|c|}
\hline & Elcatonin Group & Placebo Group & \multicolumn{2}{|c|}{ Difference } \\
\hline Age (years) & $71.5 \pm 2.2$ & $72.7 \pm 2.1$ & 0.688 & $-7.4,5.0$ \\
\hline BMD at lumbar spine $\left(\mathrm{g} / \mathrm{cm}^{2}\right)$ & $0.751 \pm 0.029$ & $0.660 \pm 0.048$ & 0.119 & $-0.025,0.207$ \\
\hline Urinary NTx (nmol BCE/mmol Cr) & $71.6 \pm 11.4$ & $59.5 \pm 7.5$ & 0.381 & $-15.8,40.1$ \\
\hline
\end{tabular}

Values are listed as the mean \pm standard error. $95 \% \mathrm{CI} ; 95 \%$ confidence interval. 
Table 3. The Weekly Mean VAS Scores from the Start of Treatment (First Week) to Five Weeks Later (Sixth Week) in the Elcatonin and Placebo Groups

\begin{tabular}{|c|c|c|c|c|c|c|c|c|c|c|}
\hline & \multirow[b]{2}{*}{ Group } & \multicolumn{6}{|c|}{ Week } & \multicolumn{3}{|c|}{ p Value } \\
\hline & & 0 & 1 & 2 & 3 & 4 & 5 & Time & Group & $\begin{array}{l}\text { Time } \\
\times \\
\text { Group }\end{array}$ \\
\hline \multirow{3}{*}{$\begin{array}{l}\text { Pain } \\
\text { at } \\
\text { rest }\end{array}$} & Elcatonin & $3.00 \pm 2.60$ & $2.56 \pm 2.28$ & $1.16 \pm 1.14$ & $0.73 \pm 1.15$ & $0.39 \pm 0.68$ & $0.20 \pm 0.35$ & $<0.001$ & 0.969 & 0.992 \\
\hline & Placebo & $2.83 \pm 2.65$ & $2.81 \pm 2.87$ & $1.03 \pm 1.31$ & $0.70 \pm 0.93$ & $0.43 \pm 0.55$ & $0.34 \pm 0.59$ & & & \\
\hline & $\begin{array}{c}\text { Difference } \\
(95 \% \mathrm{CI})\end{array}$ & $\begin{array}{c}0.17 \\
(-1.73,2.07)\end{array}$ & $\begin{array}{c}-0.24 \\
(-2.14,1.65)\end{array}$ & $\begin{array}{c}0.13 \\
(-0.77,1.02)\end{array}$ & $\begin{array}{c}0.03 \\
(-0.72,0.79)\end{array}$ & $\begin{array}{c}-0.03 \\
(-0.48,0.41)\end{array}$ & $\begin{array}{c}-0.14 \\
(-0.50,0.22)\end{array}$ & & & \\
\hline \multirow{3}{*}{$\begin{array}{c}\text { Pain } \\
\text { at } \\
\text { motion }\end{array}$} & Elcatonin & $6.91 \pm 1.93$ & $4.62 \pm 1.89$ & $3.06 \pm 2.05$ & $1.83 \pm 1.84$ & $0.77 \pm 0.83$ & $0.38 \pm 0.49$ & $<0.001$ & 0.037 & 0.542 \\
\hline & Placebo & $7.04 \pm 2.44$ & $5.47 \pm 3.28$ & $4.87 \pm 2.76$ & $3.03 \pm 3.09$ & $2.05 \pm 2.18$ & $1.77 \pm 2.09$ & & & \\
\hline & $\begin{array}{c}\text { Difference } \\
(95 \% \mathrm{CI})\end{array}$ & $\begin{array}{c}-0.13 \\
(-1.74,1.48)\end{array}$ & $\begin{array}{c}-0.86 \\
(-2.86,1.14)\end{array}$ & $\begin{array}{c}-1.81 \\
(-3.59,-0.02)\end{array}$ & $\begin{array}{c}-1.20 \\
(-3.09,0.69)\end{array}$ & $\begin{array}{c}-1.28 \\
(-2.53,-0.02)\end{array}$ & $\begin{array}{c}-1.40 \\
(-2.57,-0.22)\end{array}$ & & & \\
\hline
\end{tabular}

Values are listed as the mean \pm standard deviation. $95 \% \mathrm{CI} ; 95 \%$ confidence interval.

estrogen can cause hyperalgesia [7]. This change in the level of genetic expression is one of the triggers for the pain accompanying postmenopausal osteoporosis.

Local ischemia in the paraspinal muscles is associated with changes in spinal alignment and participates in lower back pain in postomenopausal women. Degenerative or traumatic changes in spinal alignment tend to cause fatigue pain while maintaining an unnatural posture. Such changes in paraspinal muscle alignment may cause local tissue ischemia, which consequently leads to localized pain [8]. The mechanism of such ischemic pain is attributed to the fact that the lack of oxygen causes an increase in lactic acid, leading to acidosis. Algesic substances such as kallikrein and bradykinin are subsequently generated, thereby causing pain.

As mentioned above, it is thought that postmenopausal women are more vulnerable to lower back pain. Some authors have reported that calcitonin is effective for relieving lower back pain in such patients and have suggested several mechanisms in regard to how calcitonin acts as an antinociceptive substance. The mechanisms of anti-nociception by calcitonin can be generally categorized as either direct central action or indirect peripheral action.

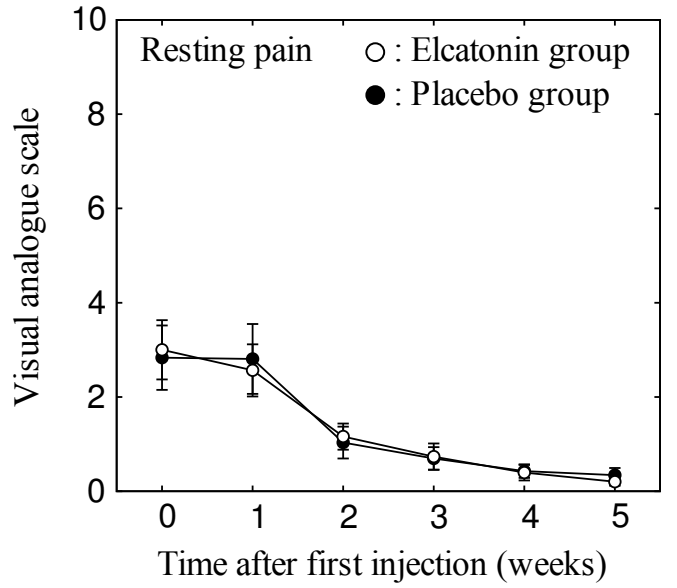

Calcitonin can act as an anti-nociceptive substance on the central nociceptive system. It is known that the central monoaminergic systems, such as the serotonergic and noradrenergic systems, contribute to the modulation of nociceptive transmission. Several reports have implicated the involvement of the serotonergic [9-11] and catecholaminergic systems [12] in calcitonin-induced antinociception. Moreover, the contribution of the serotonergic system to the anti-nociceptive effects of peripherally injected calcitonin has been suggested. Several authors have reported that elcatonin injection increases the level of spinal serotonergic receptor expression in ovariectomized rats and is also involved in antinociception $[13,14]$.

Furthermore, calcitonin may clinically contribute to pain relief through the improvement of blood flow. Nagai et al. reported that calcitonin improves blood flow in the legs and raises skin temperature $[15,16]$. Accordingly, calcitonin may increase blood flow systemically as well as in the legs. However, Brain et al. reported that the vasodilatory effects of calcitonin are weak [17], and therefore the mechanisms of increased blood flow by calcitonin remain unclear. If the injection of calcitonin leads to increased blood flow in the paraspinal muscles, it may be possible to flush out algesic

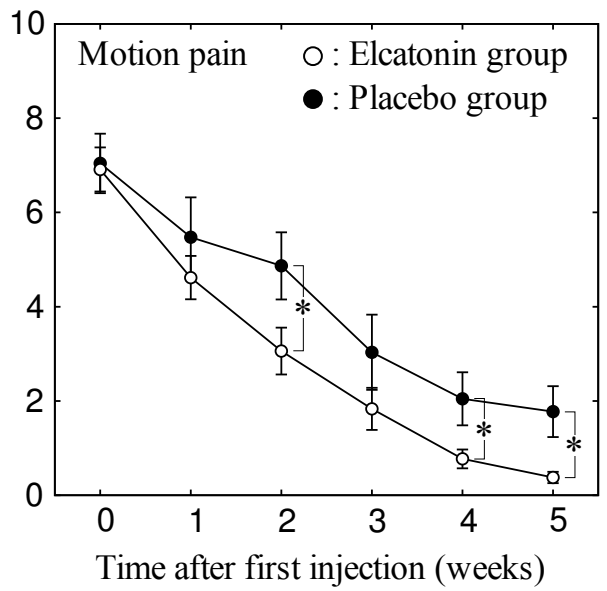

Fig. (2). The extent of pain after the first injection of elcatonin or placebo. The means of the visual analogue scale value for each group are shown. Error bars indicate the standard error. ${ }^{*} \mathrm{p}<0.05$. 
substances and to relieve ischemic or fatigue pain.

We formed a hypothesis about the analgesic effect of elcatonin injection in postmenopausal women. Acute lower back pain that is so severe as to prevent movement is typically due to vertebral compression fractures in osteoporotic patients [18]. These fractures are recognized and diagnosed in only one third of the patients who suffer from them [19]. While this condition is not commonly associated with back pain, some vertebral fractures can cause various degrees of back pain in elderly patients. Therefore, unrecognized vertebral fractures may be involved in even moderate back pain in elderly women [20]. Patients who had back pain due to such unrecognized vertebral fractures were allowed to participate in this trial. A limitation of our study is the lack of identification of such fractures in the participants of this trial because of the non-performance of MRI or scintigraphy. Chronic lower back pain, which primarily occurs at motion, is thought to be caused by articular facets, ligaments, muscle, and fascia connected with vertebral deformities after fractures in postmenopausal osteoporotic women. Pain in the muscle and fascia is thought to be fatigue pain or ischemic pain. Elcatonin may relieve such pain by increasing blood flow in the early phase of treatment.

Furthermore, elcatonin may cause pain relief in postmenopausal women by controlling hyperalgesia caused by calcitonin insufficiency due to estrogen insufficiency. Therefore, the anti-nociceptive effects of elcatonin may persist for some time after stopping treatment.

We demonstrated that the injection of elcatonin (20 units) relieves motion pain in the lower back in postmenopausal women. This injection is often performed in postmenopausal osteoporotic women with lower back pain. We propose that elcatonin should be administered to patients who complain of motion pain in their lower back. If this calcitonin drug is appropriately used in such patients, it will effectively relieve pain.

\section{REFERENCES}

[1] Foster GV, MacIntyre I, Pearse AGE. Calcitonin production and the mitochondrion-rich cells of the dog thyroid. Nature 1964; 203 : 1029-30.

[2] Pearse AGE. The cytochemistry of the thyroid C cells and their relationship to calcitonin. Proc R Soc Lond Biol 1966; 164: 478-87.

[3] Austin LA, Heath H. Calcitonin: Physiology and pathophysiology. N Engl J Med 1981; 304: 269-78.

[4] Prato A, Scapagnini U, Rapisarda E, et al. Analgesic activity of a synthetic analogue of eel-calcitonin and its effect on the spomtaneous activity of brainstem neurons in the rat. Neurosci Lett 1987; 81: 303-8.

[5] Knopp JA, Diner BM, Blitz M, Lyritis GP, Rowe BH. Calcitonin for treating acute pain of osteoporotic vertebral compression fractures: a systematic review of randomized, controlled trials. Osteoporos Int 2005; 16(10): 1281-90.

[6] Yoshikawa S, Shiba M, Hoshino T, et al. [Effect of eel calcitonin derivative (elcatonin) in osteoporosis] Nippon Seikeigeka Gakkai Zasshi 1983; 57(11): 1717-28. In Japanese.

[7] Forman LJ, Tingle V, Estilow S, Cater J. The response to analgesia testing is affected by gonadal steroids in the rat. Life Sci 1989; 45(5): 447-54.

[8] Mense S. Pathophysiology of low back pain and the transition to the chronic state - experimental data and new concepts. Schmerz 2001; 15(6): 413-7. (In German).

[9] Clementi G, Prato A, Conforto G, Scapagnini U. Role of serotonin in the analgesic activity of calcitonin. Eur J Pharmacol 1984; 98: 449-51.

[10] Clementi G, Amico-Roxas M, Rapisarda E, et al. The analgesic activity of calcitonin and the central serotonergic system. Eur J Pharmacol 1985; 108: 71-5.

[11] Colado MI, Ormazabal MJ, Goicoechea C, Lopez F, Alfaro MJ, Martin MI. Involvement of central serotonergic pathways in analgesia elicited by salmon calcitonin in the mouse. Eur $J$ Pharmacol 1994; 252: 291-7.

[12] Guidobono F, Netti C, Sibilia V, Olgiati VR, Pecile A. Role of catecholamines in calcitonin-induced analgesia. Pharmacology 1985; 31: 342-8.

[13] Yoshimura M. Analgesic mechanism of calcitonin. J Bone Miner Metab 2000; 18: 230-3.

[14] Ito A, Kumamoto E, Takeda M, Shibata K, Sagai H, Yoshimura M. Mechanisms for ovariectomy-induced hyperalgesia and its relief by calcitonin: participation of 5-HT1A-like receptor on $\mathrm{C}$-afferent terminals in substantia gelatinosa of the rat spinal cord. J Neurosci 2000; 20(16): 6302-8.

[15] Nagai T, Sakamoto K, Miyaoka H. Three cases in which a calcitonin (elcatonin) improved blood flow of the legs. J East Jpn Orthop Traumatol 2006; 18: 493-7. In Japanese.

[16] Nagai T, Sakamoto K, Miyaoka H. Effect of calcitonin for ADL in patients who have glucocorticoid-induced osteoporosis: A case report. Jpn J Rehabi Med 2003; 40: 863-8. (In Japanese).

[17] Brain SD, Williams TJ, Tippins JR, Morris HR, MacIntyre I. Calcitonin gene-related peptide is a potent vasodilator. Nature 1985; 313: 54-6.

[18] Takahara K, Kamimura M, Nakagawa H, Hashidate H, Uchiyama $\mathrm{S}$. Radiographic evaluation of vertebral fractures in osteoporotic patients. J Clin Neurosci 2007; 14(2): 122-6.

[19] Vogt TM, Ross PD, Palermo L et al. Vertebral fracture prevalence among women screened for the Fracture Intervention Trial and a simple clinical tool to screen for undiagnosed vertebral fractures. Fracture Intervention Trial Research Group. Mayo Clin Proc 2000; 75(9): 888-96.

[20] Kamimura M, Uchiyama S, Takahara K, Hashidate H, Kawaguchi A, Nakagawa H. Urinary excretion of type I collagen cross-linked $\mathrm{N}$-telopeptide and serum bone-specific alkaline phosphatase analysis to determine the correlation of age and back-pain related changes in elderly women. J Bone Miner Metab 2005; 23(6): 495 500 . 